\title{
Evaluation of Concordance Among Different Drug Information Sources Regarding the Recommendations for Dosage Adjustment in Renal Impairment
}

Kyung Hee Choi ${ }^{1,6}$, Young-Mi Ah², Soo-in Sun ${ }^{3}$, Juyun Kim ${ }^{4}$, Jinjoo Lee ${ }^{5}$, Sooyoung Shin ${ }^{3}$, Ju-Yeun Lee ${ }^{2}$ and Sukhyang Lee ${ }^{3 *}$

${ }^{1}$ College of Pharmacy, Sunchon National University, Suncheon, South Korea

${ }^{2}$ College of Pharmacy, Institute of Pharmaceutical Science and Technology, Hanyang University, Ansan, South Korea

${ }^{3}$ College of Pharmacy, Ajou University, Suwon, South Korea

${ }^{4}$ Department of Biomedical Science, College of Medicine, Seoul National University, Seoul

${ }^{5}$ Florida Hospital East Orlando, Orlando, FL

${ }^{6}$ College of Pharmacy, Chonnam National University, Gwang-Ju, South Korea

\begin{abstract}
Background: It is very important to prescribe appropriate drugs and adjust medication and dosage for optimal disease management not only to maximize patient outcomes, but also to minimize adverse events. So, the accuracy of drug information and consistency among different resources would have a significant effect on drug therapy.

Objectives: We aimed to evaluate the concordance among five drug information sources regarding the recommendations for dosage adjustment in renal impairment.

Methods: The five drug information resources were selected the Korean National Formulary Drug (KNF), the British national formula 2013 (BNF 65), Daily Med in USA, Lexicomp-online, and Drug Prescribing in Renal Failure (DPRF, 5th ed.). After having classified the degrees of renal function and defined the terms used for the dosing methods, we analyzed the concordance in dosing recommendations in renal deficiency among the information sources by calculating Fleiss Kappa.

Results: In the dosing adjustments of patients with renal insufficiency, there were some cases of contraindicated drugs that were not matched in each reference. Statistical analysis of the concordance of four references, except DPRF, and evaluation of 168 of the drugs that were mentioned in all four sources showed the Fleiss Kappa coefficientto be 0.243 . This indicatesfair agreement among the data.

Conclusion: Fair agreement was observed among 4 sources. This may be owing to discrepancies in the frequency of updating drug information sources. We suggest that the quantitative standard be used to classify renal function and to maintain consistency in definition of renal impairment.
\end{abstract}

\section{Introduction}

Pharmacological therapy is one of the important options for disease management. It is very important that the occurrence of adverse events be minimized whilemaximizing the therapeutic effect of medication[1]. Therefore, the prescribers need to use appropriate medications and dosage according to the overall patient status; present illness, underlying disease, liver or kidney function, drug allergy, etc.

The kidney plays a major role in the excretion of drugs and their metabolites.Thus, renal dysfunction can affect the pharmacokinetics of many drugs and some of these drugs may accumulate and cause toxic effects in patients with renal impairment [1]. Moreover, it has been reported that acute or chronic renal insufficiency can affect the morbidity and mortality rates of patients, especially hospitalized patients [2]. For this reason, dose adjustment according to kidney function is very important to achieve the proper effects and to avoid drug toxicity. For example, ranitidine, an $\mathrm{H}_{2}$ blocker, has been associated with serious central nervous system (CNS) adverse effectsin patients with renal insufficiency $[1,3,4]$. Imipenem/cilastatin was also associated with an increased risk of seizures in patients with renal insufficiency [1]. In other reports, when the dosage of this medicine was adjusted according to renal function, the incidence of convulsion decreased [5]. Kidney function is generally estimated using parameters such as serum creatinine $(\mathrm{Scr})$, glomerular filtration rate (GFR), and creatinine clearance (Ccr), so it was needed to have the standard of guideline according to renal function index.

When clinicians prescribe medications and adjust the dosage to suit patients with renal insufficiency, they generally consult various sources of informationsuch as other experienced health care providers or drug information resources [2,6]. Therefore, the reliability of drug information available as reference is very important, including information on contraindications and warnings or dosage reductions for special disease states. In recent years, a new order decision system called the Clinical Decision Support System (CDSS) has been adapted in hospitals. In an effort to maintain patient safety and provide highquality medical services, the incidence of drug-induced adverse events should be prevented. The use of the CDSS can optimize the selection of appropriate dosage and reduce these medication-related adverse events. For these reasons, the information provided by the CDSS needs to be accurate and consistent among various drug information sources.

However, Salgado et al. [7] analyzed and assessed the clinical relevance of the method of dose adjustment in patients with renal

"Corresponding Author: Dr. Sukhyang Lee, College of Pharmacy, Ajou University, Woncheon-dong, Yeongtong-gu, Suwon 16499, South Korea: E-mail: suklee@ajou.ac.kr

Citation: Choi KH, Ah YM, Sun SI, Kim J, Lee J, et al. (2016) Evaluation of Concordance Among Different Drug Information Sources Regarding the Recommendations for Dosage Adjustment in Renal Impairment. Int J Clin Pharmacol Pharmacother 1: 103. doi: https://doi.org/10.15344/2456$3501 / 2016 / 103$

Copyright: ( 2016 Choi et al. This is an open-access article distributed under the terms of the Creative Commons Attribution License, which permits unrestricted use, distribution, and reproduction in any medium, provided the original author and source are credited. 
Citation: Choi KH, Ah YM, Sun SI, Kim J, Lee J, et al. (2016) Evaluation of Concordance Among Different Drug Information Sources Regarding the Recommendations for Dosage Adjustment in Renal Impairment. Int J Clin Pharmacol Pharmacother 1: 103. doi: https://doi.org/10.15344/2456-3501/2016/103

Page 2 of 4

insufficiency for the medications that were approved by the European Medicines Agency (EMA) with the Summaries of Product Characteristics (SmPCs) in Europe, and they concluded that only $48.8 \%$ of them provided information that was both explicit and relevant [7].

This study aimed to evaluate the concordance among five drug information sources regarding the recommendations for dosage adjustment in renal impairment for medications that are approved by the Korean Drug Formulary (KDF) in South Korea.

\section{Methods}

We selected the drugs that required dosage adjustment in reduced renal function from among the medicines approved by the Ministry of Food and Drug Safety (MFDS) in 2011 [8]. In this process, we used three references: drugs listed as requiring renal function monitoring by the Department of Veterans ' Affairs of Australia[9], the Infectious Diseases Management Program of University of California, San Francisco (UCSF) [10], and the clinician's Ultimate Reference by Global RPh., which is one of the widely used online sources [11]. Additionally, categorized groups such as angiotensin receptor inhibitors (ACEIs), angiotensin receptor blockers (ARBs), and benzodiazepines were replaced by the specific active constituent according to other references (Appendix 1).

For the systemic comparison of the selected medications, we used five drug information sources including the KDF, the British national formulary 2013 (BNF 65), which is the main pharmaceutical reference in the United Kingdom (UK) [12], Daily Med that is operated by the U.S. National Library of Medicine (NLM), easily accessible online, and publishes up-to-date and accurate drug labels, and only one brand-name drug which selected in random was analyzed [13], and the Lexicomp-online, which is a clinical information solution. It provides a wide range of drug information and the labeled indications of medications especially those available in the USA and Canada [14] Well established as the premier source of information about drug use in patients with renal insufficiency, Drug Prescribing in Renal Failure (DPRF, 5th ed.) by Bennett et al. was also used [15].

After drug selection, we reviewed and evaluated the methods of dose recommendations in renally impaired patients from these five references. Previously, Vidal et al. and Khanal et al. had described six categories of dose adjustment methods $[9,16]$ and we modified their categories. Using this modified classification, we compared the consistency of information and its presentation in each of the five sources.

The six categories and the method of judgment was as follows [9]:

- Contraindicated (CI): This category included the drugs that were not recommended for use in patients with a specificdegree of renal impairment. We included the following statements: "contraindications administration", "contraindication", "do not administer", and "should be avoided" in the reference,but we excluded the statement "avoid" which had means not "contraindication" but "caution".

- $\quad$ Not listed (L): This category included the drugs that were not listed in the drug information sources. First, we only included the drugs in the KNF, and in case of drugs with salts, we distinguished them from their salts. This category had zero items in the KNF,because we only included the drugs.
- $\quad$ Numerical recommendations $(\mathrm{N})$ : This category included the drugs with dose adjustment data according to quantified renal scales such Ccr, GFR, and Scr. In principle, kidney function was presented in the smallest unit, but we allowed a range from normal function to $5 \mathrm{~mL} / \mathrm{min}$ in case of Ccr.

- Non-numerical recommendations $(\mathrm{NN})$ : This category included the drugs which had ambiguous dose adjustment data according to qualitative renal scales using terms such as "mild", "moderate", "severe", "nephropathy", "renal impairment", "renal failure", "renal dysfunction", and "end-stage renal failure" instead of quantified scales. It also contained the drugs with recommendations which were not further clarified with specific numericaldata, only using terms such as "with caution", "administered cautiously", or "dosage reduction".

- No advice mentioned (X): This category included the drugs which were listed in the references, but had no specific recommendations for dosage adjustment in renal impairment. This group included drugs without any information in the SmPC for dose adjustment in renal impairment.

- Dosage adjustment not required $(\mathrm{Y})$ : This category included the drugs where no dosage adjustment is needed or the recommended dosage in renal impairment was the same as in patients with no renal impairment.

We analyzed and evaluated all of the aforementioned five references with respect to the presence of dosage adjustment information according to kidney functional level. Finally, in the 215 drugs, the concordance of the references was determined by Fleiss kappa (K) coefficient using the R X64 3.1.1 program [9]. This is a statistical measurement, which evaluates the reliability of agreement between raters when assigning categorical ratings to a number of items or classifying items.It is thereforea useful tool to measure of the reliability of multiple determinations on the same subjects $[17,18]$.

Additionally, we classified the method of expressing renal function. There are two methods to express kidney status: one is a quantitative method using numerical values such as Ccr, GFR, or Scr (A, B, C), the other is a qualitative method using verbal expression or no mention related to renal function (D, XI). In addition, we defined another group where the reference omitted any information of drugs (MI).

A. This group represented renal function as Ccr.

B. This group represented renal function as GFR.

C. This group represented renal function as Scr.

D. This group represented renal function as verbal sentences.

XI. This group made no mention of renal function in dosage information.

MI. This group contained no information of drugs in the reference.

Assessment of the five references was performed by two reviewers, and a final reevaluation was undertaken.

\section{Results}

From drugs listed as requiring renal function monitoring by three references, 267 drugs were identified, and 215 drugs were included in the study after excluding 52 drugs that were not registered in the KDF. Of the 215 drugs that were analyzed for dosage adjustment owing to reduced renal function according to the Anatomical Therapeutic Chemical Classification System (ATC) by the World Health Organization (WHO), 91 drugs (42.3\%) were found to be antiinfectives for systemic use, 41 (19.1\%) were nervous system drugs, 
Citation: Choi KH, Ah YM, Sun SI, Kim J, Lee J, et al. (2016) Evaluation of Concordance Among Different Drug Information Sources Regarding the Recommendations for Dosage Adjustment in Renal Impairment. Int J Clin Pharmacol Pharmacother 1: 103. doi: https://doi.org/10.15344/2456-3501/2016/103

Page 3 of 4

\begin{tabular}{|l|l|l|l|l|l|l|l|l|l|l|l|l|l|l|}
\hline $\begin{array}{l}\text { ATC } \\
\text { Classification }\end{array}$ & A & B & C & D & G & J & L & M & N & P & R & S & $\begin{array}{l}\text { No } \\
\text { classification }\end{array}$ \\
\hline $\begin{array}{l}\text { Numbers of } \\
\text { drug }\end{array}$ & 11 & 5 & 36 & 2 & 8 & 91 & 1 & 8 & 41 & 1 & 6 & 3 & 2 \\
\hline$\%$ & 5.12 & 2.33 & 16.7 & 0.93 & 3.72 & 42.3 & 0.47 & 3.72 & 19.1 & 0.47 & 2.79 & 1.4 & 0.93 \\
\hline
\end{tabular}

Table 1: Drugs with dosage adjustment in renal failure.

A : Alimentary tract and metabolism, B : Blood and blood forming organs C : Cardiovascular system D : Dermatological drugs G :

Genitourinary system and reproductive hormones $\mathrm{J}$ : Antiinfectives for systemic use L : Antineoplastic and immunomodulating agents $\mathrm{M}$ :

Musculoskeletal system N : Nervous system P : Antiparasitic products, insecticides and repellents R : Respiratory system S : Sensory organs

and 36 (16.7\%) were cardiovascular drugs. Two of the 215 drugs in the KNF were not assigned any classification in the ATC (Table 1).

The active constituent's of 215 drugs were evaluated across five references and categorized into the six modified categories (Table 2). In the $\mathrm{L}$ category, there were $40(19 \%)$ and 71 cases (33\%) of missing drug information in the BNF and DPRF respectively; there were only 5 cases $(2.3 \%)$ of missing drug information in Lexicomp. In terms of accuracy, 40 cases of CI and 66 cases of $\mathrm{N}$ categories in the KNF (106 cases out of 215 (49\%) had clear information on dosage guidelines in renal impairment. Another national formulary, the BNF, amounted to 89 drugs $(41 \%)$ in the CI category that clearly expressed nominal kidney function. Above all, Lexi-comp showed 143 of a total 215 drugs where dosage adjustment is needed according to quantified renal functions. It therefore provided the greatest amount of information among the five references.

\begin{tabular}{|l|l|l|l|l|l|}
\hline Category & KNF & $\begin{array}{l}\text { Lexi- } \\
\text { comp }\end{array}$ & $\begin{array}{l}\text { Daily } \\
\text { med }\end{array}$ & BNF & DPRF \\
\hline Contraindicated(CI) & 40 & 17 & 3 & 6 & 1 \\
\hline Not Listed(L) & 0 & 5 & 20 & 40 & 71 \\
\hline $\begin{array}{l}\text { Numeric } \\
\text { recommendation (N) }\end{array}$ & 66 & 126 & 96 & 83 & 103 \\
\hline $\begin{array}{l}\text { Non-numeric } \\
\text { recommendation } \\
\text { (NN) }\end{array}$ & 97 & 37 & 39 & 70 & 0 \\
\hline No advice (X) & 9 & 15 & 53 & 15 & 0 \\
\hline Not required (Y) & 3 & 15 & 4 & 1 & 40 \\
\hline Total drugs & 215 & 215 & 215 & 215 & 215 \\
\hline
\end{tabular}

Table 2: Information provided for the renal dosing drugs.

KNF: Korean National Formulary, BNF: British National Formulary, DPRF: Drug Prescribing in Renal Failure

The $\mathrm{K}$ coefficient was used to determine the concordance among the five references. For statistical calculation of kappa coefficient, the raters, subjects, and categories of the data were defined as follows: raters $(\mathrm{m})$ were the references of drug information, subjects $(\mathrm{N})$ were drugs that did not have missing data in any references, and categories (к) were methods of dose recommendation. For objective analysis of the data, we analyzed only drugs that had data in all references, so we compared the data of 122 subjects, including categories CI, N, NN $\mathrm{X}$, and Y,and excluding category $\mathrm{L}$ in all five references. As a result, we calculated the kappa coefficient to be 0.196 , and concluded that there is a slight agreement between the five raters. DPRF has been the preeminent drug information resource providing dosage adjustment guidelinesin cases of declined kidney function. Since its publication in 2007, several drugs have been developed, and incomplete updating of recently developed formulations has been a limitation. When we excluded DPRF and assessed only the remaining four references, the $\mathrm{K}$ coefficient turned out to be 0.243 . This was judged to be a fair level of concordance $[17,19]$. The result was comparable to a $\mathrm{K}$ coefficient of 0.258 , which shows fair level of concordance between Lexi-comp, Daily Med, and the BNF. Consequently, we determined that the four references, excluding DPRF, had a fair level of concordance (Figure 1).

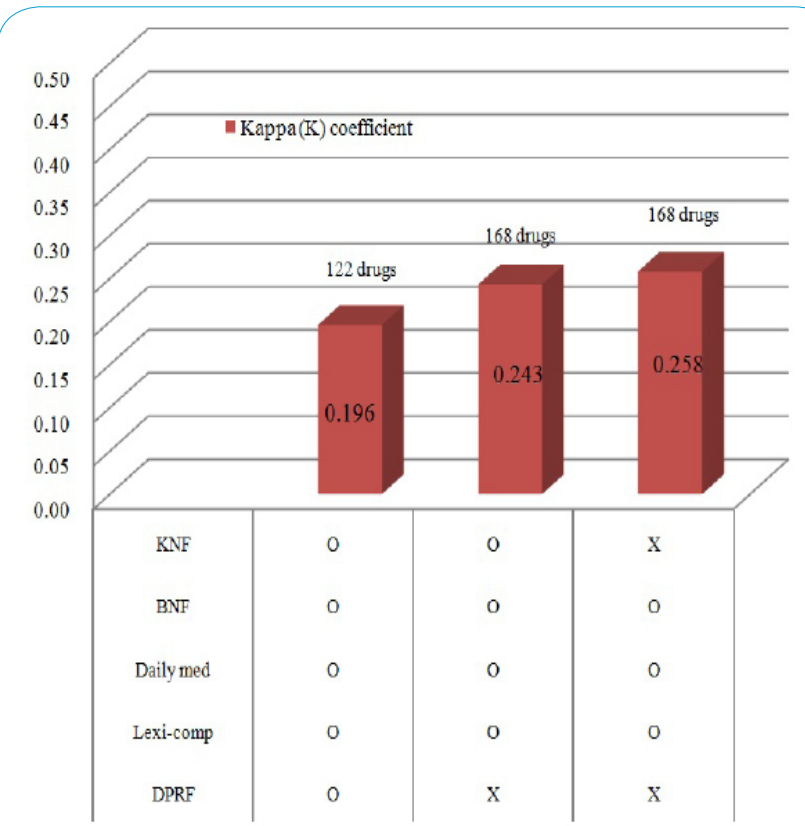

Figure 1: Kappa coefficient of the concordance among references.

In the expression of renal function, the KNF used nominal renal functional values like Ccr, GFR, and Scr in 120 cases (56\%) with function mainly expressed according to Ccr (47\%). Linguistic expression totaled 86 cases (40\%) represented by terms such as 'renal impairment' and 'kidney dysfunction' to describe renal function and words such as 'mild,' 'moderate', and 'severe' to represent the severity of impairment. The term 'contraindication' was also included in this category. Moreover, nine cases $(4 \%)$ in the KNF had no dosage adjustment information according to decreased renal function.

\section{Discussion}

Recently, large databases housing various information on drugs have been developed and increasingly used in medical prescription to avoid medication misuse. For example, CDSS is the representative supporting system in clinical practice andcan provide varied information on appropriate medical treatments according to the patient's status. It can therefore help the practitioners in improving their prescribing patterns and accuracy. In addition, it could help to enhance communication between the clinicians and other supporting 
Citation: Choi KH, Ah YM, Sun SI, Kim J, Lee J, et al. (2016) Evaluation of Concordance Among Different Drug Information Sources Regarding the Recommendations for Dosage Adjustment in Renal Impairment. Int J Clin Pharmacol Pharmacother 1: 103. doi: https://doi.org/10.15344/2456-3501/2016/103

departments. During the course of treatment, medication errors were found to occur mainly during prescription; hence, it is of great significance that such prescription errors could be prevented by CDSS [20]. Furthermore, a well designed web-based computerized tool expected the well support the decision-making processin various disease status [21]. CDSS can be used within computerized programs and has been generally usedalong with computerized physician order entry (CPOE) systems such as order communication systems (OCS) or electronic medical records (EMR) [22]. CDSS, when used in combination with these systems can potentially provide a wealth of information including drug interactions, therapeutic duplication, dosage adjustment in renal impairment, etc. Therefore, keeping the contents accurate and concise is an important consideration when configuring these systems.

According to a report by Chertow et al., when the CPOE system was used to guide medication dosing for inpatients with renal insufficiency, both the quality of prescription, and theadherence to guidelines improved [2]. Based on these results, we recommended the adaptation of dosage datain certain medications that require dosage modification in accordance with the change of renal function. It was necessary to verify the consistency of the adapted data with different sources to examine the accuracy of the supplied information. The KNF is an authoritative reference in South Korea and it plays an important role in providing drug information. In this paper, we compared KNF with other drug information references including BNF, Daily Med, DPRF, and Lexi-comp to determine concordance regarding the dosing recommendations for drugs that required careful dosage adjustment in renal impairment. We originally sorted 267 active constituents into 12 ATC categories based on literature review. Among them 215 ingredients were finally chosen as listed in the KNF. Then, each drug in each reference was evaluated for the quality of information. In the KNF, all the drugs except forninetypes of components were recommended for modification of dosage according to kidney status. However, several drugs had recommendations written in vague expressions instead of quantitative values of renal function and dosage. There should be an improvement in the method of presentation of information for these drugs [1]. For example, digoxin is widely known to need dosage adjustment in kidney dysfunction, as administration without appropriate dose reduction can lead to serious adverse reactions including AV block, nevertheless, only vague phases were used for dosage adjustment in the KNF such as "carefully administered" and "easy to cause poisoning in low dose" [8].The other three references used quantitative values, and in the case of the BNF the method of dosage adjustment was elucidated with phrases such as "reduce dose and monitor plasma digoxin concentration; toxicity increased by electrolyte disturbance" [12]. Moreover, 40 cases were presented as contraindications in the KNF, making it the largest number of drugs among the 5 references. The KNF, however, used non-numerical expressions in 13 of 40 cases, in comparison to Lexicomp, which had only 3 of 23 cases $[8,14]$.

The concordance in information within the references is very important, as accurate and consistent information is essential to the medical decision-making process, especially in special situations like renal dysfunction. Kappa coefficient is a method for judging the degree to which the raters agree, so we used it for examining the agreement among the references.

In this study, the standards set by Landis and Koch (1977) were adapted for the determination of concordance in data among several references [17]. When the calculated kappa coefficient is $0.21-0.40$, it would indicate a fair degree of concordance. However, the occurrence of missing groups that have no data in the specific references was approximately $19 \%$ in the BNF and 33\% in DPRF. These missing entries could influence the outcome of the test; we therefore decided to exclude the BNF, which had the most number of missing drugs. Finally, 168 active constituents, except the missing groups in the four references, were assessed and the resulting kappa coefficient, 0.243 , indicated fair agreement. Another kappa coefficient that examined the three references Lexi-comp, Daily Med, and the BNF, was 0.258 , which also shows a fair degree of agreement. As shown in Table 3 we found that quantitative expressions of the kidney function accounted for a relatively high proportion. Adjusting the dosage according to the nonnumeric data is known to be difficult. Therefore, it has been suggested that standardized expressions of kidney status should be used in drug information. When using quantitative expressions, we could decide the dosage according to the standardized descriptions of renal function. Ryu et al. has outlined the difficulties in interpreting diverse representations of kidney function and in recommending appropriate drug use[6].

\begin{tabular}{|l|l|l|l|l|l|}
\hline Category $^{*}$ & KNF & Lexi-comp & Daily med & BNF & DPRF \\
\hline A & 112 & 147 & 104 & 8 & 2 \\
\hline B & 1 & 5 & 5 & 103 & 142 \\
\hline C & 7 & 4 & 1 & 2 & 0 \\
\hline D & 86 & 39 & 32 & 47 & 0 \\
\hline MI & 0 & 5 & 20 & 40 & 71 \\
\hline XI & 9 & 15 & 53 & 15 & 0 \\
\hline Total drugs & 215 & 215 & 215 & 215 & 215 \\
\hline${ }^{*}$ It counted 'A' in 3 cases of used both 'A', 'C', and 'B' in 2 cases of used \\
\hline
\end{tabular}

*It counted 'A' in 3 cases of used both 'A', 'C', and 'B' in 2 cases of used both 'B', 'C'

Table 3: Presentation of renal function for dosing recommendation.

A. creatine clearance (Ccr), B. glomerular filtration rate (GFR), C. serum creatinine (Scr) D. lingual expression with renal dysfunction state, MI. no information of drug, XI. no dosage guideline according to renal function in drug information.

One limitation of this study is that different sources of drug information update their own data at different time intervals. It was therefore impossible to compare between drug information in different sources at exactly the same point in time. At the same time, the study results highlighted the importance of periodic updates in drug information that we need to use in a medical environment. In the case of the BNF, drug information is revised and updated every six months. We believe that similar regular updating and publishing is required for the KNF.

\section{Conclusion}

In this study, we investigated the concordance of dosage adjustment in patients with renal insufficiency across the representative drug information references including Lexi-comp, Daily Med, the BNF, DPRF, and the KNF. We concluded that there was fair agreement based on the calculated kappa coefficient between different resources except in the case of DPRF. This was owing to differences in the evidence data and the update cycle of drug information.

In conclusion, it is necessary to require periodic updates of drug information and to present specific criteria of drug use in specific patient populations (such as those with kidney dysfunction) in a concrete, accurate, and concordant fashion in drug information resources, especially when working with CDSS. 
Citation: Choi KH, Ah YM, Sun SI, Kim J, Lee J, et al. (2016) Evaluation of Concordance Among Different Drug Information Sources Regarding the Recommendations for Dosage Adjustment in Renal Impairment. Int J Clin Pharmacol Pharmacother 1: 103. doi: https://doi.org/10.15344/2456-3501/2016/103

Page 4 of 4

\section{Competing Interests}

The author(s) declare that they have no competing interests.

\section{Author Contributions}

Conception and design: Kyung Hee Choi, Juyun Kim, Soo-young Shin Analysis and interpretation: Kyung Hee Choi, Young-Mi Ah

Data collection: Young-Mi Ah, Soo-in Sun

Writing the article: Kyung Hee Choi

Critical revision of the article: Jinjoo Lee, Ju-Yeun Lee

Final approval of the article: Sukhyang Lee

Statistical analysis: Kyung Hee Choi

Obtained funding: Sukhyang Lee

Overall responsibility: Sukhyang Lee

\section{Acknowledgement}

This study received funding from the Ministry of Health \& Welfare, Reublic of Korea.The sponsor did not participate in the study design, data collection or interpretation. Only the authors approved the final manuscript.

\section{Funding}

This study was supported by a grant of the Korea Healthcare R\&D Project, the Ministry of Health \& Welfare, Republic of Korea (Grant No. HI13C0723).

\section{References}

1. Choi N, Lee JY, Cho YS, Kim HS, Son IJ, Lee HS (2011) Assessing prescribing behavior in hospitalized patients with renal impairment. J Kor Soc Health-Syst Pharm 28:13.

2. Chertow GM, Lee J, Kuperman GJ, Burdick E, Horsky J, Seger DL, et al. (2001) Guided medication dosing for inpatients with renal insufficiency. JAMA 286: 2839-2844.

3. Karras DJ (1994) Severe low back pain secondary to acute interstitial nephritis following administration of ranitidine. The American journal of emergency medicine.12: 67-68.

4. Slugg PH, Haug MT, Pippenger CE (1992) Ranitidine pharmacokinetics and adverse central nervous system reactions. Arch Intern Med 152: 23252329 .

5. Calandra G, Lydick E, Carrigan J, Weiss L, Guess H (1988) Factors predisposing to seizures in seriously ill infected patients receiving antibiotics: experience with imipenem/cilastatin. The American journal of medicine 84: 911-918.

6. Ryu JH, Kyoung EJ, Lee HY, Oh M, Kim EY (2012) Comparative Analysis of Drug InformationResources for Dose Adjustment in terms of Renal and Hepatic Function. Kor J Clin Pharm 22.

7. Salgado TM, Arguello B, Martinez-Martinez F, Benrimoj SI, FernandezLlimos F (2013) Clinical relevance of information in the Summaries of Product Characteristics for dose adjustment in renal impairment. European journal of clinical pharmacology. 69: 1973-1979.

8. Korean National Formulary, 2011.

9. Khanal A, Castelino RL, Peterson GM, Jose MD (2013) Dose adjustment guidelines for medications in patients with renal impairment: how consistent are drug information sources? Intern Med J 44: 77-85.

10. The Regents of the University of California, 2014

11. David McAuley PD, The Clinician's Ultimate Reference.

12. BNF (2013) British National Formulary 65.

13. DailyMed: U.S. the National Library of Medicine; 2014.

14. Lexicomp-online (2013) Wolters Kluwer Health.

15. Swan SK, Bennett WM (2007) Drug dosing guidelines in patients with renal failure. West J med 156

16. Vidal L, Shavit M, Fraser A, Paul M, Leibovici L (2005) Systematic comparison of four sources of drug information regarding adjustment of dose for renal function. BMJ 331: 263.
17. Landis JR, Koch GG (1977) The Measurement of Observer Agreement for Categorical Data. Biometrics 33: 159-174.

18. http://en.wikipedia.org/wiki/Fleiss\%27_kappa

19. Zaiontz C (2014) Real Statistics Using Excel.

20. Phillips DP, Christenfeld N, Glynn LM (1998) Increase in US medicationerror deaths between 1983 and 1993. Lancet 351: 643-644.

21. Franchi D, Cini D, lervasi G (2011) A new Web-based medical tool for assessment and prevention of comprehensive cardiovascular risk. Ther Clin Risk Manag 7: 59-68.

22. Seo J, Lee E, Lee J, Cho K, Ham J (2010) Evaluation of Clinical Decision Supporting System as part of the Order Communication System. J Kor Soc Health-Syst Pharm 27. 